\title{
Mesenteric Lymphatic Malformation Causing Midgut Volvulus in an Adult: An Unusual Presentation
}

\author{
Surya Nandan Prasad ${ }^{1} \quad$ Rani Kunti R. Singh ${ }^{1} \quad$ Pragya Chaturvedi ${ }^{1} \quad$ Vivek Singh ${ }^{1}$ \\ ${ }^{1}$ Department of Radiodiagnosis, Sanjay Gandhi Post Graduate \\ Institute of Medical Sciences, Lucknow, Uttar Pradesh, India \\ Address for correspondence Surya Nandan Prasad, MD, PDCC, \\ Department of Radiodiagnosis, Sanjay Gandhi Post Graduate \\ Institute of Medical Sciences, Lucknow 226014, Uttar Pradesh, India \\ (e-mail: drsurya1@gmail.com).
}

J Gastrointestinal Abdominal Radiol ISGAR 2021;4:243-246.

\begin{abstract} Keywords

- lymphatic malformation

- mesentery

- midgut volvulus

- whirlpool sign

- bowel obstruction
\end{abstract}

\section{Introduction}

Lymphatic malformations are benign lesions of vascular origin showing differentiation in the line of lymphatic tissue. Common locations are head, neck, and axilla during childhood and less than $5 \%$ lesions occur in mesentery, retroperitoneum, lung, and mediastinum. Mesentery is the most common site for intraperitoneal lymphatic malformation and may result in complications like volvulus, intestinal obstruction, and even infarction. Being a nonfixed mobile structure, a large mesenteric lesion can undergo torsion and exert traction over the mesentery. This may lead to rotation of mesentery along with small bowel loop resulting in midgut volvulus and closed loop bowel obstruction. Midgut volvulus is a well-known entity in infants and children but rare in adults and often complicated with intestinal obstruction. Here we are presenting a case of large multicystic lymphatic malformation of mesentery causing midgut volvulus in an adult with classical radiological findings.

\section{Case Presentation}

A 20-year-old man visited routine outpatient department (OPD) with complaints of a palpable mass in lower abdomen and occasional pain and abdominal distension. The mass was first noticed by the patient $\sim 3$ years ago and since it was asymptomatic, he did not seek medical help before. In last 2 months the patient had three episodes of colic pain and abdominal distension which subsided on conservative management. On examination the patient's vitals were normal and a palpable mass was noted in central and right lower abdomen. His routine blood investigations were within the normal limit. Ultrasonography (USG) of abdomen showed a large heteroechoic mass with multiple small cystic areas within, occupying central and right lower quadrant of abdomen ( - Fig. 1). The lesion appeared to arise within the mesentery, displacing adjacent small bowel loops without any sign of bowel obstruction. No significant intralesional vascularity was noted on color Doppler USG. Further evaluation published online April 26, 2021
DOI https://doi.org/ $10.1055 / \mathrm{s}-0041-1727581$ ISSN 2581-9933 (c) 2021. Indian Society of Gastrointestinal and Abdominal Radiology. This is an open access article published by Thieme under the terms of the Creative Commons Attribution-NonDerivative-NonCommercial-License, permitting copying and reproduction so long as the original work is given appropriate credit. Contents may not be used for commercial purposes, or adapted, remixed, transformed or built upon. (https://creativecommons.org/licenses/by-nc-nd/4.0/). Thieme Medical and Scientific Publishers Pvt. Ltd. A-12, 2nd Floor, Sector 2, Noida-201301 UP, India 
with contrast-enhanced computed tomography (CECT) showed a large well-demarcated hypodense nonenhancing mesenteric lesion occupying central abdomen and extending to right iliac fossa (RIF) ( - Fig. 2). Small bowel loops were seen wrapping around its periphery. There was no evidence of abnormal bowel wall thickening, communication between bowel lumen and the mass or signs of bowel obstruction. Lymphatic malformation was kept as provisional diagnosis and elective magnetic resonance imaging (MRI) abdomen was planned for better preoperative evaluation of the mass. The patient again presented in emergency department after 2 weeks with pain in abdomen, distension, and two episodes of vomiting. MRI evaluation ( - Fig. 3) showed a large well-demarcated T2 hyperintense multicystic lesion with multiple T2 hypointense thin septations. On postcontrast scan, minimal enhancement of septations was noted. There was twisting of small bowel loop with its mesentery at the level just cranial to the mass, with whirling of the mesenteric vessels and small bowel around the superior mesenteric artery ( - Fig. 4). A dilated small bowel loop was seen tapering with a beaked appearance toward the center of the whirl and continued distally to the twisted and collapsed small bowel loop abutting the cystic mass. Superior part of the cystic mass was insinuated into the whirl. Proximal small bowel loops and stomach were dilated and distended with fluid. Whirling of the mesenteric vessels and small bowels around the superior mesenteric artery were also noted on color Doppler USG. All these findings were suggestive of volvulus of the small bowel with closed loop obstruction caused by torsion of the large multicystic mesenteric mass.

\section{Treatment and Outcome}

Emergency laparotomy was performed and a large lobulated mass arising from the small bowel (ileal) mesentery was found at $90 \mathrm{~cm}$ proximal to ileocecal junction. There was torsion of the mass that caused $360^{\circ}$ volvulus of the ileal loops and connecting mesentery, resulting in closed loop small bowel obstruction. The volvulus was reduced by derotating the mass and the mass was completely excised along with resection of adjacent small bowel segment (18 cm length).

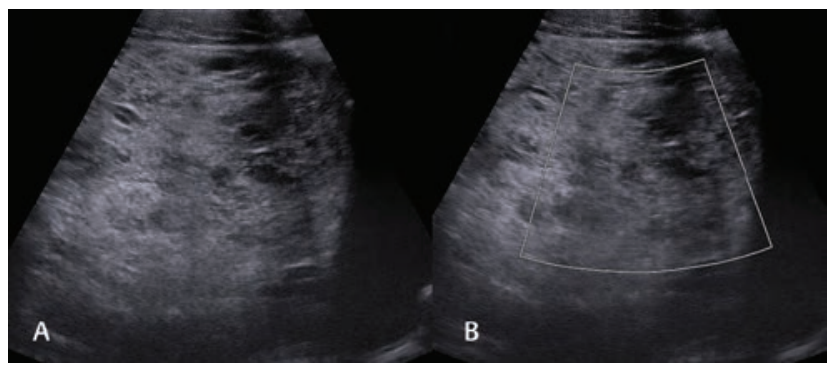

Fig. 1 Ultrasonography (USG) performed at initial presentation. (A) Gray-scale USG image showing a heteroechoic mass with multiple small cystic areas within. (B) Color Doppler image shows absence of vascularity within the mass.

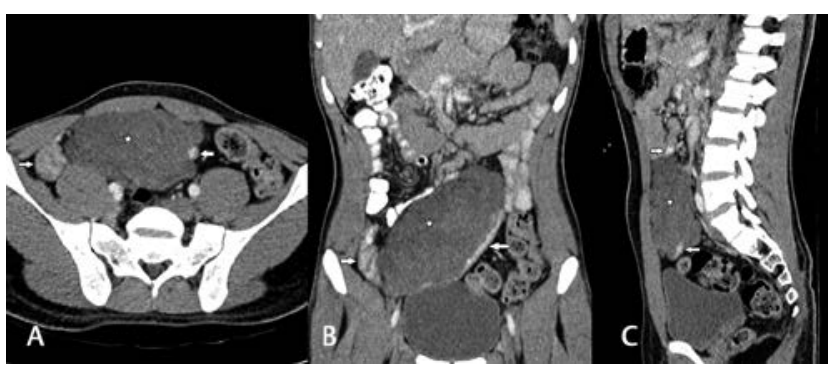

Fig. 2 Computed tomography (CTO scan performed at initial presentation. Contrast-enhanced CT scan in (A) axial, (B) coronal, and (C) sagittal planes showing a large lobulated hypodense mesenteric mass (asterisk) occupying lower abdomen and right iliac fossa. Small bowel loops filled with oral contrast (arrows) are seen wrapping around the lesion.

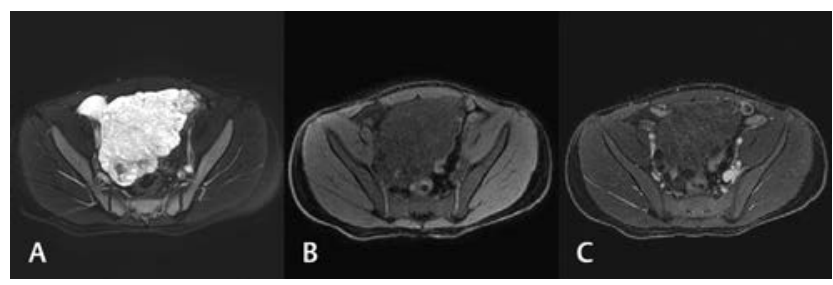

Fig. 3 Axial magnetic resonance imaging (MRI) images performed 2 weeks after initial presentation. (A) T2-weighted image (T2WI) with fat-saturated (FS) sequence showing a well-defined hyperintense lobulated multicystic mass occupying lower abdomen and pelvis. On (B) pre- and $(\mathbf{C})$ post-contrast FS T1WI images, the mass is hypointense and show minimal thin septal enhancement.

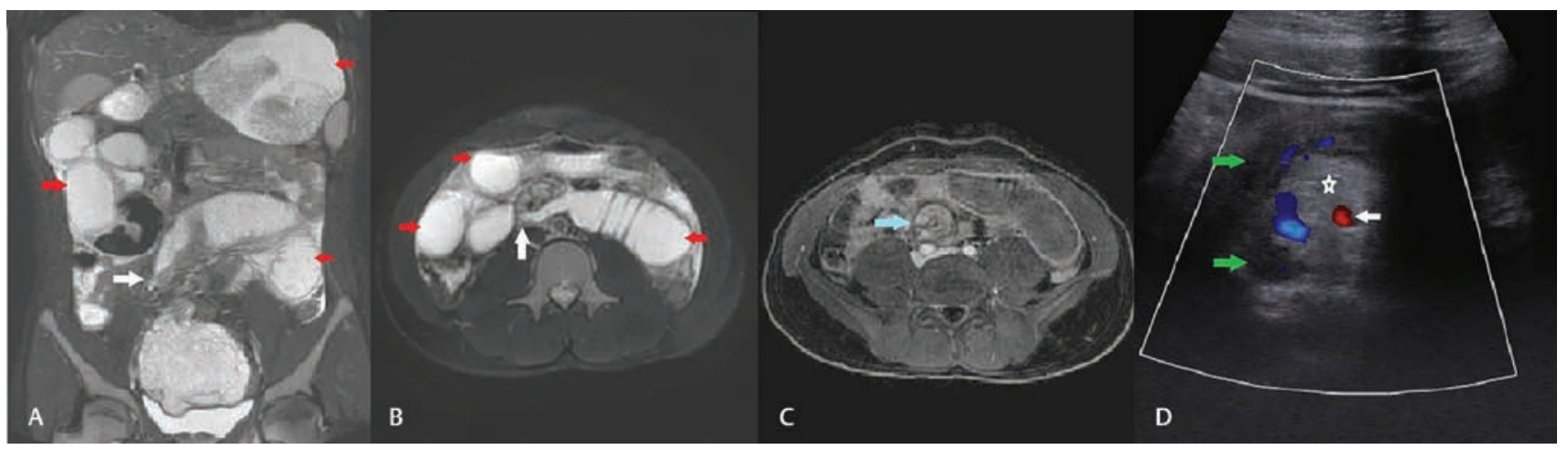

Fig. 4 Magnetic resonance imaging (MRI) and color Doppler images performed 2 weeks after initial presentation. (A) Coronal fat-saturated (FS) T2-weighted image (T2WI) shows tapering and beaking (white arrow) of a dilated small bowel loop cranial to the multicystic mass with dilated proximal small bowel loops and stomach (red arrows). (B) Axial FS T2WI and (C) postcontrast FS T1WI at the level of transition point cranial to multicystic mass shows tapering of bowel lumen and twisted small bowel loop around a central vascular axis, giving MRI equivalent of classical "whirlpool sign." (D) Color Doppler image showing twisted small bowel loop (green arrows) and its mesentery (asterisk) around a central vascular axis (white arrow) giving classical "whirlpool sign." The central vascular axis is formed by superior mesenteric artery. 
Grossly the mass was encapsulated with bosselated surface, which on cutting open showed multiple cystic spaces filled with clear fluid. Histopathological evaluation confirmed it as lymphatic malformation. There was no surgical complication. Post surgery, the patient recovered uneventfully; he was discharged on the postoperative day 7 and advised to come for follow-up in routine OPD.

\section{Discussion}

Midgut volvulus is characterized by torsion of the bowel loop along with its mesentery around a central vascular pedicle. Small bowel volvulus can be attributed to either primary or secondary causes. Primary midgut volvulus occurs without any predisposing factor or anatomical defect. There are multiple predisposing factors for secondary midgut volvulus including bowel malrotation, postoperative adhesions, congenital bands, intussusception, fistula, tumors, and Meckel's diverticulum. ${ }^{1}$ The most common cause of pediatric midgut volvulus is bowel malrotation. ${ }^{2}$

Lymphatic malformations are benign lesions of vascular origin. They are considered congenital malformations and result due to aberration in development of vascular tissue. Sometimes lymph containing cystic lesions may form due to obstruction of lymphatic vessels after trauma, surgery, any inflammatory process, or radiotherapy. ${ }^{3}$ Lymphatic cystic lesions developing secondary to lymphatic vessel obstruction usually result in unilocular cysts without septations. ${ }^{4}$ The commonly used term "lymphangioma" to describe these lesions in the past is abolished now because they do not represent true neoplasm of the lymphatic system. ${ }^{5}$ The most common locations are head, neck, and axilla during childhood, which account for $>95 \%$ cases. Remaining $5 \%$ cases occur in mesentery, retroperitoneum, lung, and mediastinum. ${ }^{6,7}$ Small bowel mesentery is the most common site for intraperitoneal lymphatic malformations with 50 to $60 \%$ of them located in the ileal mesentery. ${ }^{8}$ Pediatric population is commonly affected and young age group favors congenital origin of the lesion.

Generally mesenteric lymphatic malformations remain asymptomatic for long durations. When symptomatic, they most commonly present with palpable abdominal mass and occasional abdominal distension. ${ }^{9}$ Bowel obstruction may be caused by compression of intestine by the large mass. Sometimes lymphatic malformation may undergo torsion along with the mesentery, resulting in complications like bowel volvulus, closed loop intestinal obstruction, or even bowel infarction..$^{10}$ Large mesenteric lymphatic malformations causing midgut volvulus and intestinal obstruction is a rare entity and needs immediate diagnosis and management. ${ }^{1,11}$ Chronic midgut volvulus with a mesenteric lymphatic malformation and underlying malrotation has also been reported in literature. ${ }^{12}$

Multicystic mesenteric lymphatic malformations are nonfixed mobile lesions and have higher chances of developing complications. So they are diagnosed earlier when compared with nonmobile retroperitoneal lymphatic malformations. Two distinct theories exist in literature to explain the association of mesenteric lymphatic malformations and midgut volvulus. The first theory states that nonfixed nature of mesenteric lymphatic malformation exerts traction over the mobile mesentery, leading to its rotation along with adjacent bowel loops, resulting in volvulus formation and closed loop bowel obstruction. According to another theory, recurrent or chronic midgut volvulus may cause lymphatic obstruction which ultimately leads to development of mesenteric lymphatic cysts. In this case the lymphatic cystic lesion will be unilocular and usually without septations. ${ }^{12,13}$ In our case, the multicystic multiseptated nature of the lesion and the fact that it was present for several years before the development of midgut volvulus support the diagnosis of congenital lymphatic malformation rather than acquired lymphatic cyst.

On cross-sectional imaging, mesenteric lymphatic malformations can be diagnosed with reasonable accuracy and complications like midgut volvulus are detected early. USG findings of mesenteric lymphatic malformation include well-circumscribed variable echogenicity multicystic mass with thin septations displacing adjacent bowel loops. USG proves better than computed tomography (CT) when it comes to characterization of the lesion like capsular thickness, septation, or echogenicity of the content. Echogenic contents within the lesion may indicate hemorrhage or secondary infections. On color Doppler evaluation these lesions are avascular; however, sometimes minimal septal vascularity may be seen. In presence of midgut volvulus, the twisted bowel loop and mesenteric vessels are very well-demonstrated in real time on color Doppler evaluation, giving typical :whirlpool"-like appearance. This "whirlpool sign" is described in literature as an objective sign of midgut volvulus and it was present in our case at the time of color Doppler evaluation. On CECT, the mesenteric lymphatic malformations appear as a well-demarcated lobulated hypodense mass. Attenuation of the lesion varies depending upon nature of the content which is usually fluid; however, sometimes it may show fat or hemorrhage within. Calcification is rare. Characteristic thin cyst walls and intracystic septations may show minimal postcontrast enhancement on CECT scan. ${ }^{11,14}$ Multiplanar reformations of CT images help in better localization of the lesion and demonstrate relationship with adjacent structures as well as in assessment of complications such as volvulus formation or bowel obstruction.

MRI having better soft tissue resolution well demonstrates the nature of the lesion, its relation to adjacent structures, and complications like hemorrhage within the lesion. Lymphatic malformations are T1 hypointense and T2 hyperintense multicystic lesions. The thin septations appear hypointense on T2-weighted images and may show minimal postcontrast enhancement. In case of midgut volvulus, the dilated and distended bowel loops are seen proximal to transition point formed by the twisted small bowel loop and its mesentery. In our case, the twisted bowel loop, its mesentery, and mesenteric vessels together gave an appearance similar to the whirlpool sign demonstrated during color Doppler and CECT evaluation. 
USG and CT features of midgut volvulus are described in literature; however, we did not find any case describing MRI appearance of midgut volvulus. This may be due to the fact that MRI examination is time consuming and midgut volvulus, being a surgical emergency, needs early diagnosis and treatment. In our case, we were able to perform MRI because the patient was already dated for the examination on his prior visit to OPD 2 weeks ago. So, in our opinion cross-sectional imaging including both CECT and MRI are highly sensitive and specific in demonstrating features of midgut volvulus and bowel obstruction. MRI has added advantage of better characterization of the culprit lesion and its relation to the adjacent structures besides nil radiation exposure.

Surgery is the treatment of choice which includes de-rotation of the volvulus and complete excision of lymphatic malformation. ${ }^{13}$ At times it is not possible to separate the mass from mesenteric vasculature or the bowel loop. In that case segmental resection of adjacent bowel loop may also be performed safely as was done in our case.

\section{Conclusions}

Lymphatic malformations are benign lesions of vascular origin, primarily affecting pediatric population and rare in adults. Mesenteric lymphatic malformations by undergoing torsion may lead to formation of midgut volvulus and closed loop small bowel obstruction. Cross-sectional imaging helps in making accurate diagnosis and "whirlpool sign" is a highly specific objective sign of midgut volvulus on cross-sectional imaging evaluation. MRI scores over CECT when it comes to evaluation of the nature of the lesion, its relation with adjacent structures, and certain complications.

\section{Funding}

None.

Conflict of Interest

None declared.

\section{References}

1 Huang JC, Shin JS, Huang YT, et al. Small bowel volvulus among adults. J Gastroenterol Hepatol 2005;20(12):1906-1912

2 Namasivayam J, Ziervogel MA, Hollman AS. Case report: volvulus of a mesenteric cyst-an unusual complication diagnosed by CT. Clin Radiol 1992;46(3):211-212

3 Roisman I, Manny J, Fields S, Shiloni E. Intra-abdominal lymphangioma. Br J Surg 1989;76(5):485-489

4 Siegel MJ, Glazer HS, St Amour TE, Rosenthal DD. Lymphangiomas in children: MR imaging. Radiology 1989;170(2): 467-470

5 Jang JH, Lee SL, Ku YM, An CH, Chang ED. Small bowel volvulus induced by mesenteric lymphangioma in an adult: a case report. Korean J Radiol 2009;10(3):319-322

6 Chen CW, Hsu SD, Lin CH, Cheng MF, Yu JC. Cystic lymphangioma of the jejunal mesentery in an adult: a case report. World J Gastroenterol 2005;11(32):5084-5086

7 Lugo-Olivieri CH, Taylor GA. CT differentiation of large abdominal lymphangioma from ascites. Pediatr Radiol 1993; 23(2):129-130

8 Chin S, Kikuyama S, Hashimoto T, Tomita T, Hasegawa T, Ohno Y. Lymphangioma of the jejunal mesentery in an adult: a case report and a review of the Japanese literature. Keio J Med 1993; 42(1):41-43

9 Ros PR, Olmsted WW, Moser RP Jr, Dachman AH, Hjermstad BH, Sobin LH. Mesenteric and omental cysts: histologic classification with imaging correlation. Radiology 1987;164(2): 327-332

10 Mar CR, Pushpanathan C, Price D, Cramer B. Best cases from the AFIP: omental lymphangioma with small-bowel volvulus. Radiographics 2003;23(4):847-851

11 Lilia BH, Rym K, Ali G, et al. Abdominal cystic lymphangioma complicated by mesenteric volvulus: a case report. Tunis Med 2014;92(5):356-358

12 Yoon HK, Han BK. Chronic midgut volvulus with mesenteric lymphangioma: a case report. Pediatr Radiol 1998;28(8):611

13 Traubici J, Daneman A, Wales P, Gibbs D, Fecteau A, Kim P. Mesenteric lymphatic malformation associated with smallbowel volvulus-two cases and a review of the literature. Pediatr Radiol 2002;32(5):362-365

14 Davidson AJ, Hartman DS. Lymphangioma of the retroperitoneum: CT and sonographic characteristic. Radiology 1990; 175(2):507-510 